\title{
ABBREVIATIONS AND METHOD OF CITATION
}

The list of abbreviations in the bibliography, pp. $448 \mathrm{f}$., applies also to the footnotes of the text.

Quotations from certain authors are cited according to standard critical editions of their works, abbreviated as follows:

Eckermann, Gespräche = Eckermann, Gespräohe mit Goethe (Leipzig, Insel Verlag, 1923); $796 \mathrm{pp}$.

Goethe, Werke = Goethe, Werke (Weimar 1887-1919); 123 vols.

Hagedorn, Werke $=$ Hagedorn, Poetische Werke, ed. J. J. Eschenburg (Hamburg 1800); 5 vols.

Haller, Gedichte $=$ Haller, Gediohte, ed. L. Hirzel (Frauenfeld 1882) (Bibliothek älterer Schriftwerke der deutschen Schweiz. ... III); dxxxvi +423 pp.

Haller, Tagebuch = Haller, Tagebuch seiner Beobachtungen über Schriftsteller und über sich selbst, ed. Heinzmann (Berne 1787); 2 vols.

Heine, Werke $=$ Heine, Sämtliche Werke, ed. O. Walzel (Leipzig 1915); 10 vols.

Herders Briefwechsel $=$ Herders Briefwechsel mit Caroline Flachsland, ed. H. Schauer, Schriften der Goethe-Gesellschaft XXXIX (1926) and XLI (1928).

Herder, Werke $=$ Herder, Sämtliohe Werke, ed. Suphan (Berlin 18771913); 33 vols.

Lessing, Schriften = Lessing, Sämtliche Schriften, ed. LachmannMuncker (Stuttgart and Leipzig 1886-1924); 23 vols.

Ludwig, Schriften = Ludwig, Gesammelte Schriften, ed. Ad. Stern and E. Schmidt (Leipzig 1891); 6 vols.

Schiller, Briefe $=$ Schiller, Briefe, ed. F. Jonas (Stuttgart etc. 1896); 7 vols.

Schiller, Werke = Schiller, Sämtliche Werke, ed. Güntter and Witkowski (Leipzig 1920ff.); 20 vols.

Wieland, Briefe = Wieland, Ausgewählte Briefe an versohiedene Freunde . . 1751-1810. . . ed. Gessner (Zürich 1815); 4 vols.

Works listed in the bibliography are cited according to their bibliographical serial number.

The elevated numeral after a bibliographical entry denotes the edition, e.g., Schmidt $[160]^{2}$ I 36 . 